\title{
Goldbach Conjecture, Twin Primes Conjecture, and Bounded Gap Theorem in the Language of Number Theory
}

\author{
Open Mathematics Collaboration*† \\ September 3, 2021
}

\begin{abstract}
We write the formulas of the theorem and the conjectures highlighted in the title of this white paper in the language of number theory for pedagogical purpose in first-order logic.
\end{abstract}

keywords: Goldbach conjecture, bounded gap theorem, number theory language, first-order logic

The most updated version of this white paper is available at https://osf.io/u45y3/download https://zenodo.org/record/5428990

\section{Introduction}

1. This is a pedagogical white paper on first-order logic.

2. Our purpose is to discuss a result in [1] which is licensed under [2].

3. We use minimal notation but preserving all relevant mathematical information.

*All authors with their affiliations appear at the end of this white paper.

†Corresponding author: mplobo@uft.edu.br|Open Mathematics Collaboration 


\section{Meta-linguistic symbols}

4. := means that what is on the left is defined by what is on the right.

5. : $\equiv$ means that the strings on both sides are identical.

\section{The language of number theory}

6.

$$
\mathcal{L}_{N T}=\{0, S,+, \cdot, E,<\}
$$

7. $0:=$ number zero

8. $S(x)=x+1:=$ successor function

9. $+, \cdot,<:=$ usual binary operations

10. $E:=$ exponentiation (e.g. $E(3,2)=9)$

11. $\mathcal{L}_{N T}:=$ the language of number theory assumed to be interpreted with respect to $\mathbb{N}=\{0,1,2,3, \ldots\}$

\section{Prime number}

12. $n \in \mathbb{N}$

13. $\bar{n}:=\underbrace{S S \ldots S S} 0$

$n$ times

14. prime $(x): \equiv \overline{1}<x \wedge \neg(\exists y)(\exists z)[(y+\overline{2}) \cdot(z+\overline{2})=x]$

15. Note that $+\overline{2}$ appears summing $y$ and $z$ in (14) due to (11).

16. $\operatorname{prime}(n)$ states that $n$ is a prime number. 


\section{There is no largest prime}

17.

$$
(\forall x)(\exists y)[x<y \wedge \text { prime }(\mathrm{y})]
$$

\section{Twin Primes Conjecture}

18. There are infinitely many pairs $(x, y)$ such that $x$ and $y$ are both prime and $y=x+2$.

19.

$$
(\forall x)(\exists y)[x<y \wedge \operatorname{prime}(y) \wedge \operatorname{prime}(y+\overline{2})]
$$

20.

$(\forall x)(\exists y)(\exists z)[x<y \wedge y<z \wedge \operatorname{prime}(y) \wedge \operatorname{prime}(z) \wedge z<y+\overline{3}]$

21. (19) and (20) are equivalent.

\section{Goldbach Conjecture}

22. Any even number, except 0 and 2, can be written as a sum of two primes.

23.

$$
(\forall x)[(\exists y)[(y+\overline{2}) \cdot \overline{2}=x] \rightarrow(\exists u)(\exists v)[\operatorname{prime}(u) \wedge \operatorname{prime}(v) \wedge u+v=x]]
$$

\section{Bounded Gap Theorem}

24. There are infinitely many pairs of prime numbers that differ by 70,000,000 or less.

25.

$(\forall x)(\exists y)(\exists z)[x<y \wedge y<z \wedge \operatorname{prime}(y) \wedge \operatorname{prime}(z) \wedge z<y+\overline{70000000}]$ 
26. This theorem was proved by Yitang Zhang in 2013 [3-5].

\section{Final Remarks}

27.

$$
\mathcal{L}_{N T}=\{0, S,+, \cdot, E,<\}
$$

28.

$$
\mathbb{N}=\{0,1,2,3, \ldots\}
$$

29.

$$
\overline{1}<x \wedge \neg(\exists y)(\exists z)[(y+\overline{2}) \cdot(z+\overline{2})=x]
$$

30.

$$
(\forall x)(\exists y)[x<y \wedge \text { prime }(\mathrm{y})]
$$

31.

$$
(\forall x)(\exists y)(\exists z)[x<y \wedge y<z \wedge \operatorname{prime}(y) \wedge \operatorname{prime}(z) \wedge z<y+\overline{3}]
$$

32.

$$
(\forall x)(\exists y)[x<y \wedge \operatorname{prime}(y) \wedge \operatorname{prime}(y+\overline{2})]
$$

33.

$$
(\forall x)[(\exists y)[(y+\overline{2}) \cdot \overline{2}=x] \rightarrow(\exists u)(\exists v)[\operatorname{prime}(u) \wedge \operatorname{prime}(v) \wedge u+v=x]]
$$

34.

$(\forall x)(\exists y)(\exists z)[x<y \wedge y<z \wedge \operatorname{prime}(y) \wedge \operatorname{prime}(z) \wedge z<y+\overline{70000000}]$

\section{Open Invitation}

Review, add content, and co-author this white paper [6,7]. Join the Open Mathematics Collaboration.

Send your contribution to mplobo@uft.edu.br. 


\section{Open Science}

The latex file for this white paper together with other supplementary files are available in $[8,9]$.

\section{How to cite this paper?}

https://doi.org/10.31219/osf .io/u45y3

https://zenodo.org/record/5428990

\section{Acknowledgements}

+ Center for Open Science https://cos.io

+ Open Science Framework https://osf.io

+ Zenodo https://zenodo.org

\section{Agreement}

All authors agree with [7].

\section{References}

[1] Leary, Christopher C., and Lars Kristiansen. A friendly introduction to mathematical logic, 2nd edition, 2015.

https://knightscholar.geneseo.edu/geneseo-authors/6

[2] CC. Creative Commons. https://creativecommons.org 
[3] IAS. "Yitang Zhang's Spectacular Mathematical Journey." https://www. ias. edu/ideas/2014/zhang-breakthrough

[4] Quanta magazine. "Unheralded Mathematician Bridges the Prime Gap." https://bit.1y/3v4WcAu

[5] Zhang, Yitang. "Bounded gaps between primes." Annals of Mathematics (2014): 1121-1174. https://bit.1y/3aryBSS

[6] Lobo, Matheus P. "Microarticles." OSF Preprints, 28 Oct. 2019. https://doi.org/10.31219/osf.io/ejrct

[7] Lobo, Matheus P. "Simple Guidelines for Authors: Open Journal of Mathematics and Physics." OSF Preprints, 15 Nov. 2019.

https://doi.org/10.31219/osf .io/fk836

[8] Lobo, Matheus P. "Open Journal of Mathematics and Physics (OJMP)." OSF, 21 Apr. 2020.

https://doi.org/10.17605/osf.io/6hzyp

https://osf.io/6hzyp/files

[9] https://zenodo.org/record/5428990

[10] COS. Open Science Framework. https://osf .io

\section{The Open Mathematics Collaboration}

Matheus Pereira Lobo (lead author, mplobo@uft.edu.br) $)^{1,2}$ https://orcid.org/0000-0003-4554-1372

${ }^{1}$ Federal University of Tocantins (Brazil)

${ }^{2}$ Universidade Aberta (UAb, Portugal) 